\title{
Decreased sirtuin 4 expression is associated with poor prognosis in patients with invasive breast cancer
}

\author{
QINGYU SHI ${ }^{1}$, TONG LIU ${ }^{1}$, XIANYU ZHANG ${ }^{1}$, JINGSHU GENG $^{2}$, XIAOHUI HE $^{3}$, MING NU $^{1}$ and DA PANG ${ }^{1}$ \\ Departments of ${ }^{1}$ Breast Surgery, ${ }^{2}$ Pathology and ${ }^{3}$ Medical Records, \\ The Third Affiliated Hospital of Harbin Medical University, Harbin, Heilongjiang 150040, P.R. China
}

Received April 13, 2015; Accepted July 7, 2016

DOI: $10.3892 / \mathrm{ol} .2016 .5021$

\begin{abstract}
Aberrant metabolism is a hallmark of human cancer. Glutamine metabolism has been identified as a central metabolic pathway in cancer and thus, targeting glutamine metabolism may exhibit therapeutic potential. Sirtuin 4 (SIRT4) is an important molecule that mediates the blockade of glutamine catabolism by inhibiting glutamate dehydrogenase. In the present study, SIRT4 protein expression levels were analyzed in 409 breast cancer tissues and 241 paired adjacent non-cancerous tissues by immunohistochemical analysis and the correlation between SIRT4 expression and the clinicopathological features was evaluated. SIRT4 protein was markedly increased in the breast cancer cells compared with adjacent non-tumor mammary cells and was correlated with estrogen receptor, progesterone receptor, nuclear-associated antigen $\mathrm{Ki}-67$ and tumor protein p53 status, as well as breast cancer subtypes. Furthermore, low SIRT4 expression was associated with poor overall survival in breast cancers patients, particularly in Luminal A patients. Univariate and multivariate analyses confirmed that increased SIRT4 expression was an independent predictive factor of good prognosis for breast cancer patients. In conclusion, SIRT4 expression represents a significant favorable prognostic factor for patients with invasive breast cancer.
\end{abstract}

\section{Introduction}

Breast cancer is one of the most common types of cancer and the leading cause of cancer-associated mortality among women, worldwide (1). At present, the incidence of breast cancer in China is increasing: Breast cancer has become the second most frequently diagnosed cancer, accounting for 248,620 novel cases in 2011, with an annual incidence rate of 37.86 cases per 100,000 individuals. In 2011, breast cancer accounted

Correspondence to: Dr Da Pang, Department of Breast Surgery, The Third Affiliated Hospital of Harbin Medical University, 150 Haping Street, Harbin, Heilongjiang 150040, P.R. China E-mail:pangda@ems.hrbmu.edu.cn

Key words: breast cancer, immunohistochemistry, sirtuin 4 protein, survival, tissue microarray analysis for 60,473 mortalities with a mortality rate of 9.21 mortalities per 100,000 individuals in China (2). An increasing number of studies have focused on the identification of novel therapeutics for the treatment of breast cancer, including novel anti-metabolic therapy (3). Aberrant metabolism is a hallmark of cancer; metabolic reprogramming of cancer cells is observed in various cancers, including breast cancer (4). Mammalian cell proliferation uses glutamine as an important anaplerotic source to replenish metabolites in the tricarboxylic acid cycle (TCA) for biosynthesis (5). Glutamine metabolism is one of the central metabolic pathways in cancer, and thus targeting glutamine metabolism may present a novel therapeutic approach for the treatment of breast cancer patients with different molecular subtypes of the disease (5-7). Glutamine is the main source for TCA anaplerosis in proliferating cells: Glutamine is firstly converted to glutamate by glutaminase (GLS) followed by conversion to $\alpha$-ketoglutarate by either glutamate dehydrogenase (GDH) or transamination-coupled reactions. Sirtuin 4 (SIRT4) directly downregulates GDH activity via adenosine diphosphate (ADP)-ribosylation, which regulates glutamine catabolism. SIRT4 is an important gene that mediates the blockade of glutamine catabolism and therefore, it is considered a 'glutamine gatekeeper' $(7,8)$.

The human SIRT4 gene is located on chromosome 12. SIRT4 is a member of the SIRT family (SIRT1-7) of protein deacetylases and ADP-ribosylases that are involved in multiple cellular processes, including the maintenance of genomic stability and regulation of metabolism (9). SIRT1, -6 and -7 are localized to the nucleus, SIRT2 resides in the cytosol and SIRT3, -4 , and -5 are usually located in the mitochondria. SIRT4 predominantly acts as an ADP-ribosyltransferase, exhibiting demalonylase, desuccinylase and deacetylase activities in specific tissues (9-11). The SIRT4 gene encodes a member of the SIRT family of proteins, which are grouped into four types that are characterized by a SIRT core domain (12). The functions of SIRT4 outside pancreatic $\beta$-cells are unknown. At present, the function of SIRT4 enzymatic activity in the mitochondrion and in nuclear gene transcription remains unclear. A recent study (10) indicated that reduced SIRT4 expression causes both increased glutamine-dependent proliferation and stress-induced genomic instability, resulting in tumorigenic phenotypes. Furthermore, SIRT4-knockout mice spontaneously develop lung tumors (10). Another study (5) revealed 
that SIRT4 mRNA expression is lower in bladder, breast, colon, stomach, ovarian and thyroid carcinomas compared with normal tissues. In addition, loss of SIRT4 mRNA expression is associated with shorter time to metastasis in breast cancer patients (5), suggesting that SIRT4 expression correlates with the development and progression of breast cancer and that targeting SIRT4 may present a therapeutic target for the treatment of the disease. However, the protein levels of SIRT4 in breast cancer and its significance remain unclear. In the present study, immunohistochemical (IHC) analysis of SIRT4 protein expression in breast cancer tissues was performed to investigate the association between SIRT4 protein expression and clinicopathological features and survival of advanced breast cancer patients.

\section{Materials and methods}

Patient samples. A total of 409 histologically confirmed breast cancer and 241 adjacent non-cancerous breast tissues obtained during surgery between January 2003 and December 2007 and assessed in the Department of Pathology of The Affiliated Tumor Hospital of Harbin Medical University (Harbin, China) were retrospectively reviewed. Patients selected in this study were aged from 28 to 76 years (median, 49.66 years) Adjacent non-cancerous breast tissues located $2 \mathrm{~cm}$ from the tumor tissue were resected. The cancer and non-cancerous breast tissues were formalin-fixed, paraffin-embedded and cut into $4-\mu \mathrm{m}$ sections for IHC analysis. The inclusion criteria were as follows: Presence of primary, unilateral and operable invasive breast cancer and available data regarding initial diagnosis and clinical follow-up. The exclusion criteria were as follows: Locally advanced disease with recurrent tumors, metastatic disease and neoadjuvant therapy. The estrogen receptor (ER), progesterone receptor (PR), human epidermal growth factor receptor 2 (HER2), tumor protein p53 (p53) and nuclear-associated antigen Ki-67 (Ki-67) expression status was analyzed in all patients; tissue sections were incubated with antibodies to the proteins ER (catalog no. TA506414; dilution, 1:150; incubation time, $20 \mathrm{~min}$; temperature, $\left.25^{\circ} \mathrm{C}\right), \mathrm{PR}$ (catalog no. TA802606; dilution, 1:150; incubation time, $10 \mathrm{~min}$; temperature, $25^{\circ} \mathrm{C}$ ), HER2 (catalog no. TA503443; dilution, 1:50; incubation time, $30 \mathrm{~min}$; temperature, $25^{\circ} \mathrm{C}$ ), p53 (catalog no. TA502780; dilution, 1:100; incubation time, $15 \mathrm{~min}$; temperature, $25^{\circ} \mathrm{C}$ ) and $\mathrm{Ki}-67$ (catalog no. TA500265; dilution, 1:50; incubation time, $20 \mathrm{~min}$; temperature, $25^{\circ} \mathrm{C}$ ) antibodies (Origene Technologies Inc., Rockville, MD, USA). Individual samples with $\geq 14 \% \mathrm{Ki}-67^{+}$tumor cells were considered to exhibit high levels of proliferation (13). The St. Gallen International Expert Consensus system was used to grade the tissues (14). The present study was approved by the Ethical Committee of Harbin Medical University and written informed consent for the analysis of tissue specimens was obtained from all patients.

Patient follow-up. All patients were followed up every 3 months post-surgery for the first 5 years and every 12 months thereafter. The follow-up was conducted at The Affiliated Hospital of Harbin Medical University and patient clinical records were periodically reviewed. In addition, the overall survival (OS) was assessed: Overall survival time was defined as the time from the initial diagnosis to the date of death or the study endpoint (December 31, 2012).
Tissue microarray (TMA) and IHC analysis. Tissue blocks containing 409 invasive breast carcinoma samples and 241 paired adjacent normal breast tissue samples were arrayed. The breast cancer TMA for each tissue sample was prepared from the paraffin blocks using a thin-walled needle (inner diameter, $2 \mathrm{~mm}$ ). All tissue blocks were cut with a microtome to $4-\mu \mathrm{m}$ and affixed to slides. IHC analysis of SIRT4 was performed using a standard two-step method (15). Tissue specimens underwent antigen retrieval in citrate buffer $(\mathrm{pH} \mathrm{6.0)}$ followed by incubation overnight at $4^{\circ} \mathrm{C}$ with polyclonal rabbit SIRT4 antibody (catalog no. ab105039; dilution, 1:150; Abcam, Cambridge, MA, USA). Secondary antibodies were of the Anti-Rabbit IgG Detection system (catalog no. SP-9001; ZSGB-Bio, Beijing, China). Slides incubated with phosphatebuffered saline and rabbit serum served as the blank and negative control groups, respectively. Positively immunostained slides were used as positive controls.

SIRT4 protein expression was only analyzed in the cytoplasm (as an indirect measure of the mitochondrial protein expression). The percentage and intensity of positive SIRT4 staining was evaluated in 10 randomly selected high-power fields (magnification, x400; Olympus BX53; Olympus, Tokyo, Japan) by two investigators blinded to the clinical characteristics and outcomes of the patients. The proportion of positively-stained tumor cells in a field was scored as follows: 0 , none; $1,<10 \% ; 2,10-50 \%$; and $3,>50 \%$. The staining intensity in a field was scored as follows: 0 , no staining; 1, weak staining appearing as a light yellow color; 2 , moderate staining appearing as a yellowish-brown color; and 3, strong staining appearing as a brown color. The staining index (SI) was calculated as follows : $\mathrm{SI}=$ average staining intensity score $\mathrm{x}$ proportion score. Cut-off values for protein expression were determined by measuring heterogeneity. Scores of $<4$ and $\geq 4$ indicated low and high SIRT4 expression, respectively (16).

Statistical analysis. All data analysis was performed using MedCalc statistical software (version 11.2.0.0; MedCalc Software, Oostende, Belgium). The $\chi^{2}$ test was used to analyze the associations between SIRT4 expression and clinicopathological features, including age, lymph node metastasis (LNM), pathological stage, ER, PR, HER2 and Ki-67 status and molecular subtype. The OS times of the different patient groups were evaluated using the log-rank test. The Cox proportional hazards model was used for univariate and multivariate regression analyses. $\mathrm{P}<0.05$ was considered to indicate a statistically significant difference.

\section{Results}

SIRT4 protein expression is significantly higher in breast cancer tissues than adjacent non-cancerous tissues. SIRT4 protein was examined in 409 breast cancer tissues and 241 paired adjacent non-tumor tissues. The cytoplasmic expression of SIRT4 was significantly higher in cancer tissues compared with adjacent non-cancerous tissues $(\mathrm{P}<0.0001)$. Among the 409 breast cancer specimens, 94 (22.98\%) tissues exhibited positive SIRT4 expression and 315 (77.02\%) tissues exhibited negative SIRT4 expression. Representative images of IHC SIRT4 staining are shown in Fig. 1. Of the 241 paired cancer and non-cancerous tissues, 48 (19.92\%) breast cancer 
A
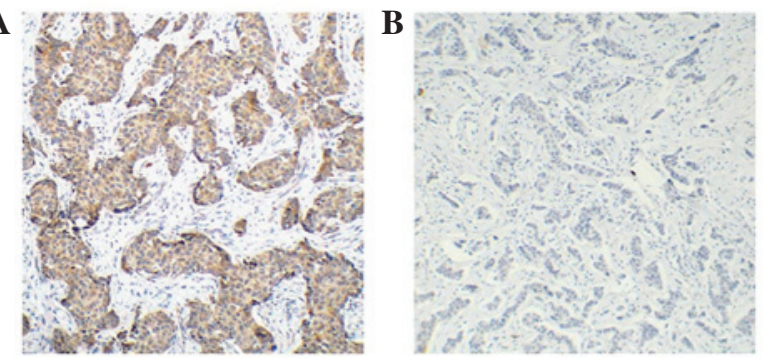

C
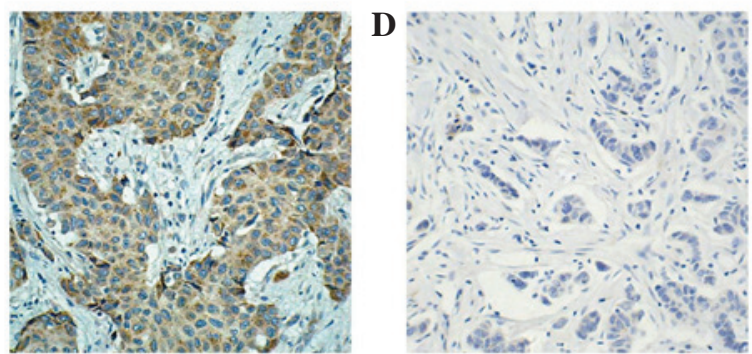

Figure 1. Representative images of SIRT4 immunohistochemical staining (A) Strong positive and (B) negative SIRT4 staining (magnification, x200x). (C) Strong positive and (D) negative SIRT4 staining (magnification, $x 400$ ). SIRT4, sirtuin 4.

tissue samples were SIRT4 positive whereas only 29 (12.03\%) adjacent non-cancerous tissue samples were SIRT4 positive $(\mathrm{P}<0.001)$.

SIRT4 expression is associated with ER, PR and p53 status and molecular subtypes of breast cancer. To investigate the association between SIRT4 expression and clinicopathological parameters, the 409 breast cancer tissue samples were divided into two subgroups based on their SIRT4 expression (negative and positive SIRT4 expression). The differences in clinical parameters, including age, LNM, TNM pathological stage and grade, ER, PR, HER2, Ki-67 and p53 status and molecular subtypes were compared between the two groups (Table I). Significant differences between the SIRT4 positive and negative expression groups were identified for the following parameters: ER status $(\mathrm{P}=0.0068), \mathrm{PR}$ status $(\mathrm{P}=0.0073), \mathrm{p} 53$ status $(\mathrm{P}=0.0040)$ and molecular subtypes $(\mathrm{P}=0.0404)$. SIRT4 protein expression rates were significantly different in the different subtypes of breast cancer $(\mathrm{P}=0.0404)$. The overall positive expression rate in each subtype was extremely low. SIRT4 protein expression rates were higher in Luminal B $(27.97 \%)$ and Luminal A $(20.00 \%)$ subtypes than the HER2 (13.73\%) and triple negative breast cancer (TNBC) (16.09\%) subtypes. Notably, the negative SIRT4 expression rates were significantly higher in TNBC $(83.91 \%)$ and HER2-positive subtypes $(86.27 \%)$ than that of the Luminal A $(72.03 \%)$ and Luminal B $(80 \%)$ subtypes $(\mathrm{P}=0.0404)$. The positive SIRT4 protein expression rates were significantly higher in ER or PR positive patients than that in ER or PR negative patients $(\mathrm{P}=0.0111)$. The expression of SIRT4 was significantly higher in the p53-positive cancer tissues compared with the p53-negative tissues $(\mathrm{P}=0.0040)$. However, no significant correlation was identified between SIRT4 expression and patient age, LNM, tumor size, TNM stage, pathological grade, HER2 status or Ki-67 status ( $\mathrm{P}>0.05$; Table I).
High SIRT4 expression is a favorable prognostic factor for breast cancer. Kaplan-Meier survival analysis was performed to investigate the association between SIRT4 expression and the survival time of patients with certain subtypes of invasive breast cancer. As shown in Table II, univariate analyses identified SIRT4 expression $(\mathrm{P}=0.0205)$, pathological stage $(\mathrm{P}=0.0019), \mathrm{PR}$ status $(\mathrm{P}=0.0127), \mathrm{Ki}-67$ status $(\mathrm{P}=0.0003)$ and LNM $(\mathrm{P}<0.0001)$ as significant prognostic predictors. The median OS time of the low SIRT4 expression group was 67.8 months, which was significantly shorter than that of the high SIRT4 expression group (73.2 months) $(\mathrm{P}=0.0205$; Fig. 2A). Multivariate analysis identified that SIRT4 expression $(\mathrm{P}=0.02985), \mathrm{Ki}-67$ status $(\mathrm{P}=0.01767)$ and $\mathrm{LNM}$ $(\mathrm{P}=0.001393)$ were independent prognostic factors (Table II). The results of this study indicate that high SIRT4 expression is a favorable prognostic factor for patients with invasive breast cancer.

Positive SIRT4 expression is associated with longer OS times in patients with Luminal A breast cancer and ER/PR positive patients. Kaplan-Meier OS curves were established to determine the effects of SIRT4 expression on survival in patients with different molecular subtypes of breast cancer. No significance differences in survival were identified between the Luminal B, HER2 and TNBC subtypes $(\mathrm{P}>0.05)$. In the Luminal A group, the OS time of patients with negative SIRT4 expression (70.35 months) was significantly shorter than that of patients with positive SIRT4 expression (73.64 months) $(\mathrm{P}=0.0382$; Fig. 2B). Similarly, in ER or PR positive patients, the OS time of patients with negative SIRT4 expression was significantly shorter than that of patients with positive SIRT4 expression, indicating that patients with positive SIRT4 expression exhibit a longer survival time than those with negative SIRT4 expression ( $\mathrm{P}=0.0275$; Fig. 2C).

\section{Discussion}

SIRT4 exhibits a crucial function in carcinogenesis as a tumor suppressor (5). The present study revealed that SIRT4 expression in breast cancer tissues was significantly higher than that in adjacent non-cancerous tissues. Univariate and multivariate analyses demonstrated that high SIRT4 expression is a protective factor in breast cancer patients. Notably, the positive expression rates of SIRT4 were low in both control and breast cancer tissues. Jeong et al (10) performed a meta-analysis to compare SIRT4 expression in human cancers and normal tissues, which demonstrated that SIRT4 mRNA expression levels were significantly lower in cancers of the bladder, breast, colon, stomach, ovarian and thyroid than normal tissue. In addition, low expression of SIRT4 mRNA was significantly correlated with early metastasis in breast cancer patients (10). In the present study, high levels of SIRT4 protein expression were not identified in the adjacent non-tumor tissues. We hypothesize that post-transcription regulation may affect SIRT4 expression and translation. Thus, further study is required to confirm this and to elucidate the possible underlying mechanisms.

The majority of previous studies regarding SIRT4 gene function have been performed in normal tissues (17-19). SIRTs are nicotinamide adenine dinucleotide+-dependent deacylases and ADP-ribosyltransferases that are involved in numerous 
Table I. Comparison of clinicopathological features between SIRT4 positive and negative breast cancer patients ( $\mathrm{n}=409$ ).

\begin{tabular}{|c|c|c|c|c|}
\hline \multirow[b]{2}{*}{ Parameter } & \multirow[b]{2}{*}{ Patients, $\mathrm{n}$} & \multicolumn{2}{|c|}{ Cytoplasmic SIRT4 expression } & \multirow[b]{2}{*}{ P-value } \\
\hline & & Negative, n (\%) & Positive, n (\%) & \\
\hline Age, years & & & & 0.3759 \\
\hline$<50$ & 223 & $176(78.92)$ & $47(21.08)$ & \\
\hline$\geq 50$ & 186 & $139(74.73)$ & $47(25.27)$ & \\
\hline Tumor size, $\mathrm{cm}$ & & & & 0.2251 \\
\hline$<2$ & 141 & $114(80.85)$ & $27(19.15)$ & \\
\hline$\geq 2$ & 268 & $201(75.00)$ & $67(25.00)$ & \\
\hline LNM & & & & 0.9020 \\
\hline Negative & 187 & $143(76.47)$ & $44(23.53)$ & \\
\hline Positive & 222 & $172(77.48)$ & $50(22.52)$ & \\
\hline TNM stage & & & & 0.1713 \\
\hline I-II & 78 & $55(70.51)$ & $23(29.49)$ & \\
\hline III & 331 & $260(78.55 \%)$ & $71(21.45)$ & \\
\hline Histological grade & & & & 0.1649 \\
\hline G1-2 & 143 & $104(72.73)$ & $39(27.27)$ & \\
\hline G3 & 266 & $211(79.32)$ & $55(20.68)$ & \\
\hline ER status & & & & 0.0068 \\
\hline Negative & 226 & $186(82.30)$ & $40(17.70)$ & \\
\hline Positive & 183 & $129(70.49 \%)$ & $54(29.51)$ & \\
\hline PR status & & & & 0.0073 \\
\hline Negative & 164 & $138(84.15)$ & $26(15.85)$ & \\
\hline Positive & 245 & $177(72.24)$ & 68 (27.76) & \\
\hline HER2 status & & & & 0.1447 \\
\hline Negative & 324 & $244(75.31)$ & $80(24.69)$ & \\
\hline Positive & 85 & $71(83.53)$ & $14(16.47)$ & \\
\hline Ki-67 status, \% & & & & 0.4060 \\
\hline$<14$ & 222 & 175 (78.83) & 47 (21.17) & \\
\hline$\geq 14$ & 187 & $140(74.87)$ & $47(25.13)$ & \\
\hline p53 status & & & & 0.0040 \\
\hline Negative & 79 & $71(89.87)$ & $8(10.13)$ & \\
\hline Positive & 330 & 244 (73.94) & $86(26.06)$ & \\
\hline Molecular subtype & & & & 0.0404 \\
\hline Luminal A & 236 & $170(72.03)$ & $66(27.97)$ & \\
\hline Luminal B & 35 & $28(80.00)$ & $7(20.00)$ & \\
\hline HER2 & 51 & $44(86.27)$ & 7 (13.73) & \\
\hline Triple negative & 87 & $73(83.91)$ & 14 (16.09) & \\
\hline ER/PR status & & & & 0.0111 \\
\hline Double negative & 94 & 73 (77.66) & $21(22.34)$ & \\
\hline Positive & 315 & $198(62.86)$ & 117 (37.14) & \\
\hline
\end{tabular}

SIRT4, sirtuin 4; LNM, lymph node metastasis; ER, estrogen receptor; PR, progesterone receptor; HER2, human epidermal growth factor receptor 2; Ki-67, nuclear-associated antigen Ki-67; p53, tumor protein p53.

cellular processes (17-19). SIRT4 inhibition increases fat oxidative capacity and mitochondrial function in liver and muscle cells (20). A limited number of genes directly interact with SIRT4, including cyclic adenosine monophosphate response element-binding protein 2 (CREB2), malonyl CoA decarboxylase and GDH $(5,18)$. In the present study, SIRT4 expression was significantly correlated with ER, PR and p53 status. Previously it has been demonstrated that SIRT4 regulates ADP-ribosylates and inhibits GDH to regulate glutamine metabolism (8). A gas chromatography-time-of-flight mass spectrometry-based metabolomics study revealed that the glutamate metabolite changes are increased in the ER-negative subtype of human 
Table II. Univariate and multivariate analysis of prognostic factors in invasive breast cancer patients.

\begin{tabular}{|c|c|c|c|c|c|c|}
\hline \multirow[b]{2}{*}{ Parameter } & \multicolumn{3}{|c|}{ Univariate analysis } & \multicolumn{3}{|c|}{ Multivariate analysis } \\
\hline & Risk ratio & $95 \% \mathrm{CI}$ & P-value & Risk ratio & $95 \% \mathrm{CI}$ & P-value \\
\hline $\begin{array}{l}\text { Age, years } \\
\geq 50 \text { vs. }<50\end{array}$ & 1.1505 & $0.7213-1.8350$ & 0.5538 & & & \\
\hline $\begin{array}{l}\text { Pathological stage } \\
\text { I-II vs. III }\end{array}$ & 4.3055 & $2.4185-7.6647$ & 0.0019 & 1.6719 & $0.5267-5.3077$ & 0.3856 \\
\hline $\begin{array}{l}\text { Tumor size, } \mathrm{cm} \\
\geq 2 \text { vs. }<2\end{array}$ & 1.0284 & $0.6303-1.6779$ & 0.9110 & & & \\
\hline $\begin{array}{l}\text { LNM } \\
\text { Positive vs. negative }\end{array}$ & 3.8147 & $2.3950-6.0759$ & 0.0001 & 3.0141 & $1.5375-5.9088$ & 0.0014 \\
\hline $\begin{array}{l}\text { Histological grade } \\
\text { G1-2 vs. G3 }\end{array}$ & 1.5313 & $0.9429-2.4870$ & 0.1086 & 1.6719 & $0.5267-5.3077$ & 0.3856 \\
\hline $\begin{array}{l}\text { ER status } \\
\text { Positive vs. negative }\end{array}$ & 0.8146 & $0.5108-1.2992$ & 0.3940 & & & \\
\hline $\begin{array}{l}\text { PR status } \\
\text { Positive vs. negative }\end{array}$ & 0.5585 & $0.3457-0.9023$ & 0.0127 & 0.7465 & $0.4476-1.2451$ & 0.2652 \\
\hline $\begin{array}{l}\text { HER2 status } \\
\text { Positive vs. negative }\end{array}$ & 2.0190 & $1.1183-3.6452$ & 0.0046 & 1.3858 & $0.8076-2.3781$ & 0.2387 \\
\hline $\begin{array}{l}\text { Ki- } 67 \text { status, } \% \\
\geq 14 \text { vs. }<14\end{array}$ & 2.3919 & $1.4961-3.8240$ & 0.0003 & 1.8769 & $1.1186-3.1492$ & 0.0177 \\
\hline $\begin{array}{l}\text { p53 status } \\
\text { Positive vs. negative }\end{array}$ & 0.6739 & $0.3708-1.2249$ & 0.1445 & & & \\
\hline $\begin{array}{l}\text { SIRT4 status } \\
\text { Positive vs. negative }\end{array}$ & 0.4480 & $0.2608-0.7696$ & 0.0205 & 0.4586 & $0.2277-0.9234$ & 0.0299 \\
\hline
\end{tabular}

SIRT4, sirtuin 4; CI, confidence interval; LNM, lymph node metastasis; ER, estrogen receptor; PR, progesterone receptor; HER2, human epidermal growth factor receptor 2; Ki-67, nuclear-associated antigen Ki-67; p53, tumor protein p53.

A

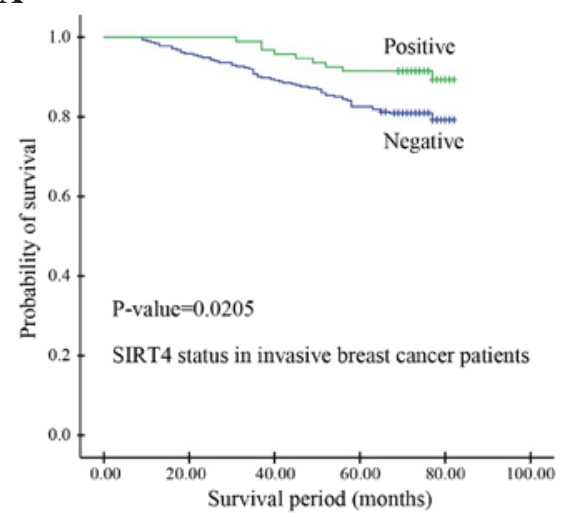

B

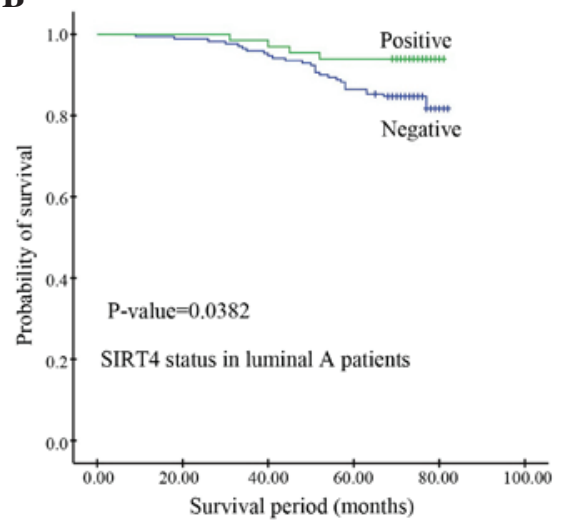

$\mathbf{C}$

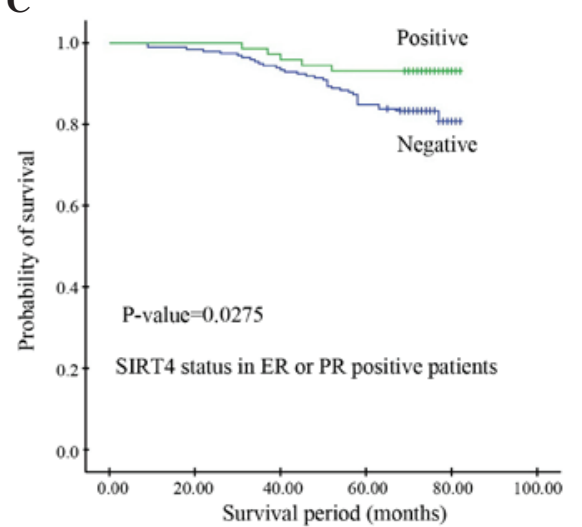

Figure 2. Kaplan-Meier survival analysis comparing OS time in patients with negative and positive SIRT4 expression. (A) Patients with negative SIRT4 expression exhibited significantly shorter survival times than those with positive SIRT4 expression $(\mathrm{P}=0.0205)$. (B) Luminal A breast cancer patients with negative SIRT4 expression exhibited shorter survival times than those with positive SIRT4 expression ( $\mathrm{P}=0.0382)$. (C) Patients with positive ER or PR status exhibited shorter survival times than those with positive SIRT4 expression $(\mathrm{P}=0.0275)$. SIRT4, sirtuin 4; ER, estrogen receptor; PR, progesterone receptor.

breast cancer (21). The detected changes included the metabolism of glutamine with a 2.2-fold decrease in the concentration of glutamine and an increase in the concentration of glutamate. The highest glutamine metabolic activity is observed in HER2 ${ }^{+}$ breast cancer and TNBC (22). In the present study, positive SIRT4 expression was identified in 54/183 (29.51\%) ER-positive patients compared with 40/226 (17.70\%) ER-negative patients. Furthermore, the OS time of patients with negative SIRT4 
expression was significantly shorter than patients with positive SIRT4 expression in ER or PR-positive and double-negative patients. ER-negative breast cancers exhibit a particular type of glutamine-dependent anaplerosis that is characterized by elevated levels of the gene encoding phosphoglycerate dehydrogenase (PHGDH) (23). Notably, inhibition of PHGDH in breast cancer cell lines induces a metabolic collapse in TCA cycle intermediates, which is also observed during DNA damage response-induced SIRT4 upregulation and GDH inhibition $(10,23)$. Thus, SIRT4 may affect the functional correlation between GDH and ER and PR.

Glutamine exhibits a critical function in cancer cell protein synthesis by supplying cellular adenosine triphosphate and serving as a metabolic intermediate for nucleotide synthesis (24). Glutamine dependence leads to growth restriction and cell death in glutamine limiting conditions, indicating that the glutamate metabolic pathway may present a potential therapeutic target (24-26). In the present study, low SIRT4 expression was associated with Luminal A breast cancer, which is a subtype of ER and PR positive breast cancer that is treated using anti-hormone receptor therapy. Among Luminal A breast cancer patients, the levels of SIRT4 expression were significantly associated with OS: Low SIRT4 expression was correlated with worse OS. In the present study, all patients with the luminal subtype of breast cancer were treated with anti-hormone receptor therapy. The drug sensitivity of anti-hormone receptors may be associated with SIRT4 expression levels. Therefore, the use of anti-hormone receptor therapy in combination with drugs that inhibit GDH or elevate SIRT4 protein levels may be beneficial in Luminal A patients. Although, no significant differences between SIRT4 expression and the OS of luminal B patients were identified, anti-HER2 therapy may affect the OS of these patients. Future studies are required to elucidate the potential association between anti-hormone drugs and SIRT4 expression.

In the present study, the positive expression rates of SIRT4 were significantly different between p53-positive and p53-negative patients. The p53 gene affects glutamine metabolism by targeting phosphate-activated mitochondrial GLS2 in tumor cells. GLS2 is a key enzyme involved in the conversion of glutamine to glutamate. It controls the apoptotic response and the cellular levels of reactive oxygen species (ROS). The tumor suppressor function of p53 may involve the interaction between glutamine metabolism, energy and ROS homeostasis (27). SIRT4 directly downregulates GDH activity via ADP-ribosylation to regulate the conversion of glutamate to $\alpha$-ketoglutaric acid (8), indicating that SIRT4 and p53 interact indirectly. However, in another study, DNA-damaging agents increased SIRT4 levels in p53-inactive HEK293T cells and p53-null human prostate cancer PC3 cells, indicating that SIRT4 is induced in a p53-independent manner. After cell DNA damage, glutamine metabolism is repressed by SIRT4 expression in the mitochondria, which leads to inhibition of cell proliferation (10).

SIRT4 exhibits a critical function in cellular metabolism by regulating mitochondrial glutamine metabolism. SIRT4 represses tumor formation in vivo. A reduction in SIRT4 expression results in larger tumors compared with controls (5). In the phosphatidylinositide 3-kinase/protein kinase B/mammalian target of rapamycin (mTOR) pathway, the mTOR complex 1 (mTORC1) gene negatively regulates the transcription of SIRT4. mTORC1 increases the binding of CREB2 to $\beta$-transducin repeat-containing protein leading to CREB2 ubiquitination (5). The transcription factor CREB2 regulates the transcription of SIRT4. The mTORC1 gene indirectly regulates SIRT4 transcription to inhibit GDH, affecting glutamine metabolism. SIRT3, -4 and -5 are all expressed in the mitochondria. SIRT3 also regulates genomic instability to repress tumorigenesis $(28,29)$. SIRT4 and -3 appear to coordinately regulate tumor cell anabolism (30).

The present study demonstrated that negative SIRT4 protein expression was an independent predictor of a worse prognosis in invasive breast carcinoma. High SIRT4 expression levels were associated with a better OS in breast cancer patients. Based on the correlation between SIRT4 protein levels and the survival status in ER and PR-positive breast cancer patients, we postulate that low SIRT4 protein expression is Luminal A patients is a more effective anti-hormone therapy when used in combination with the glutamine metabolic inhibitor. Future study is required to determine the molecular mechanism by which SIRT4 regulates the progression of breast cancer.

\section{Acknowledgements}

This study was supported by the National Natural Science Foundation of China (grant no. 81172498/H1622).

\section{References}

1. Siegel R, Naishadham D and Jemal A: Cancer statistics, 2012. CA Cancer J Clin 62: 10-29, 2012.

2. Chen W, Zheng R, Zeng H, Zhang S and He J: Annual report on status of cancer in China, 2011. Chin J Cancer Res 27: 2-12, 2015.

3. Bange J, Zwick E and Ullrich A: Molecular targets for breast cancer therapy and prevention. Nat Med 7: 548-552, 2001.

4. Kung HN, Marks JR and Chi JT: Glutamine synthetase is a genetic determinant of cell type-specific glutamine independence in breast epithelia. PLoS Genet 7: e1002229, 2011.

5. Csibi A, Fendt SM, Li C, Poulogiannis G, Choo AY, Chapski DJ, Jeong SM, Dempsey JM, Parkhitko A, Morrison T, et al: The mTORC1 pathway stimulates glutamine metabolism and cell proliferation by repressing SIRT4. Cell 153: 840-854, 2013.

6. Fernandez-Marcos PJ and Serrano M: Sirt4: The glutamine gatekeeper. Cancer Cell 23: 427-428, 2013.

7. DeBerardinis RJ, Lum JJ, Hatzivassiliou G and Thompson CB: The biology of cancer: Metabolic reprogramming fuels cell growth and proliferation. Cell Metab 7: 11-20, 2008.

8. Haigis MC, Mostoslavsky R, Haigis KM, Fahie K, Christodoulou DC, Murphy AJ, Valenzuela DM, Yancopoulos GD, Karow M, Blander G, et al: SIRT4 inhibits glutamate dehydrogenase and opposes the effects of calorie restriction in pancreatic beta cells. Cell 126: 941-954, 2006.

9. Sebastián C, Satterstrom FK, Haigis MC and Mostoslavsky R: From sirtuin biology to human diseases: An update. J Biol Chem 287: 42444-42452, 2012

10. Jeong SM, Xiao C, Finley LW, Lahusen T, Souza AL, Pierce K, Li YH, Wang X, Laurent G, German NJ, et al: SIRT4 has tumor-suppressive activity and regulates the cellular metabolic response to DNA damage by inhibiting mitochondrial glutamine metabolism. Cancer Cell 23: 450-463, 2013.

11. He W, Newman JC, Wang MZ, Ho L and Verdin E: Mitochondrial sirtuins: Regulators of protein acylation and metabolism. Trends Endocrinol Metab 23: 467-476, 2012.

12. Vassilopoulos A, Fritz K S, Petersen DR and Gius D: The human sirtuin family: Evolutionary divergences and functions. Hum Genomics 5: 485-496, 2011

13. Cheang MC, Chia SK, Voduc D, Gao D, Leung S, Snider J, Watson M, Davies S, Bernard PS, Parker JS, et al: Ki67 index, HER2 status, and prognosis of patients with luminal B breast cancer. J Natl Cancer Inst 101: 736-750, 2009. 
14. Goldhirsch A, Wood WC, Coates AS, Gelber RD, Thurlimann B and Senn HJ; Panel members: Strategies for subtypes - dealing with the diversity of breast cancer: Highlights of the St. Gallen International Expert Consensus on the Primary Therapy of Early Breast Cancer 2011. Ann Oncol 22: 1736-1747, 2011

15. Shaoqiang C, Yue Z, Yang L, Hong Z, Lina Z, Da P and Qingyuan Z: Expression of HOXD3 correlates with shorter survival in patients with invasive breast cancer. Clin Exp Metastasis 30: 155-163, 2013.

16. Liu T, Zhang X, Shang M, Zhang Y, Xia B, Niu M, Liu Y and Pang D: Dysregulated expression of Slug, vimentin, and E-cadherin correlates with poor clinical outcome in patients with basal-like breast cancer. J Surg Oncol 107: 188-194, 2013.

17. Lombard DB, Tishkoff DX and Bao J: Mitochondrial sirtuins in the regulation of mitochondrial activity and metabolic adaptation. Handb Exp Pharmacol 206: 163-188, 2011.

18. Laurent G, German NJ, Saha AK, de Boer VC, Davies M, Koves TR, Dephoure N, Fischer F, Boanca G, Vaitheesvaran B, et al: SIRT4 coordinates the balance between lipid synthesis and catabolism by repressing malonyl CoA decarboxylase. Mol Cell 50: 686-698, 2013.

19. Houtkooper RH, Pirinen E and Auwerx J: Sirtuins as regulators of metabolism and healthspan. Nat Rev Mol Cell Biol 13: 225-238, 2012

20. Nasrin N, Wu X, Fortier E, Feng Y, Bare' OC, Chen S, Ren X, Wu Z, Streeper RS and Bordone L: SIRT4 regulates fatty acid oxidation and mitochondrial gene expression in liver and muscle cells. J Biol Chem 285: 31995-32002, 2010.

21. Budczies J, Brockmöller SF, Müller BM, Barupal DK, Richter-Ehrenstein C, Kleine-Tebbe A, Griffin JL, Orešič M, Dietel M, Denkert C and Fiehn O: Comparative metabolomics of estrogen receptor positive and estrogen receptor negative breast cancer: Alterations in glutamine and beta-alanine metabolism. J Proteomics 94: 279-288, 2013.
22. McGuirk S, Gravel SP, Deblois G, Papadopoli DJ, Faubert B, Wegner A, Hiller K, Avizonis D, Akavia UD, Jones RG, et al: PGC-1 $\alpha$ supports glutamine metabolism in breast cancer. Cancer Metab 1: 22, 2013

23. Possemato R, Marks KM, Shaul YD, Pacold ME, Kim D, Birsoy K, Sethumadhavan S, Woo HK, Jang HG, Jha AK, et al: Functional genomics reveal that the serine synthesis pathway is essential in breast cancer. Nature 476: 346-350, 2011.

24. Wise DR and Thompson CB: Glutamine addiction: A new therapeutic target in cancer. Trends Biochem Sci 35: 427-433, 2010.

25. Tennant DA, Durán RV and Gottlieb E: Targeting metabolic transformation for cancer therapy. Nat Rev Cancer 10: 267-277, 2010.

26. DeBerardinis RJ and Cheng T: Q's next: The diverse functions of glutamine in metabolism, cell biology and cancer. Oncogene 29: 313-324, 2010.

27. Suzuki S, Tanaka T, Poyurovsky MV, Nagano H, Mayama T, Ohkubo S, Lokshin M, Hosokawa H, Nakayama T, Suzuki Y, et al: Phosphate-activated glutaminase (GLS2), a p53-inducible regulator of glutamine metabolism and reactive oxygen species. Proc Natl Acad Sci USA 107: 7461-7466, 2010.

28. Kim HS, Patel K, Muldoon-Jacobs K, Bisht KS, Aykin-Burns N, Pennington JD, van der Meer R, Nguyen P, Savage J, Owens KM, et al: SIRT3 is a mitochondria-localized tumor suppressor required for maintenance of mitochondrial integrity and metabolism during stress. Cancer Cell 17: 41-52, 2010.

29. Finley LW, Carracedo A, Lee J, Souza A, Egia A, Zhang J, Teruya-Feldstein J, Moreira PI, Cardoso SM, Clish CB, et al: SIRT3 opposes reprogramming of cancer cell metabolism through HIF1 $\alpha$ destabilization. Cancer Cell 19: 416-428, 2011.

30. Kumar S and Lombard DB: Mitochondrial sirtuins and their relationships with metabolic disease and cancer. Antioxid Redox Signal 22: 1060-1077, 2015 